\title{
COREOGRAFÍAR LA EXPERIENCIA URBANA: RSVP CYCLES, DE ANNA \& LAWRENCE HALPRIN
}

\section{Eneko Lorente Bilbao}

Universidad del País Vasco/Euskal Herriko Unibertsitatea. Dpto. Comunicación Audiovisual y Publicidad

\section{Resumen}

A lo largo de la segunda mitad del pasado siglo, las ciudades industriales fueron objeto de profundos procesos de regeneración y revitalización urbana, en un contexto de creciente globalización y fractura social. En los años sesenta, antes de que se manifestara la crisis estructural de la década posterior, el arquitecto y paisajista Lawrence Halprin, junto con la bailarina y coreógrafa contemporánea Anna Halprin, desarrollaron una singular metodología interdisciplinar de investigación, denominada RSVP Cycles, con la que trataban de dar respuesta a la progresiva desestructuración y pérdida de sentido de la experiencia del espacio urbano. El acrónimo RSVP Cycles alude a un procedimiento crítico de diseño de espacios urbanos basado en el estudio exhaustivo de los recursos presentes en el lugar, seguido de la propuesta de itinerarios orientados por partituras, semejantes a las notaciones coreográficas, cuya evaluación desemboca en intervenciones y performances con las que se pretende una sensibilización y re-significación de la experiencia urbana.

\section{Palabras clave: DISEÑO URBANO; COREOGRAFÍA; TRANSFORMACIÓN URBANA; RSVP CYCLES}

\section{CHOREOGRAPHING THE URBAN EXPERIENCE: RSVP CYCLES, BY ANNA \& LAWRENCE HALPRIN}

\section{Abstract}

Throughout the second half of the last century, industrial cities were the subject of profound processes of urban regeneration and revitalization, in a context of growing globalization and social fracture. In the sixties, before the manifestation of the structural crisis of the subsequent decade, the architect and landscape architect Lawrence Halprin, together with the contemporary dancer and choreographer Anna Halprin, developed a unique interdisciplinary research methodology, called RSVP Cycles, with which they tried to respond to the progressive fragmentation, destructuration and loss of meaning of the experience of urban space. The acronym RSVP Cycles refers to a critical procedure for designing urban spaces based on an exhaustive study of the resources in the place, followed by the proposal of scores, similar to choreographic notations, whose evaluation leads to interdisciplinary interventions and performances, in order to sensitize and re-signify the urban experience.

Keywords: URBAN DESIGN; CHOREOGRAPHY; URBAN TRANSFORMATION; RSVP CYCLES

\footnotetext{
Lorente Bilbao, Eneko. 2020. "Coreografíar la experiencia urbana: RSVP Cycles, de Anna \& Lawrence Halprin”. AusArt 8 (2): 89-99. D0I: 10.1387 /ausart.22042
}

\section{AUSART}




\section{INTRODUCCIÓN}

Lawrence Halprin, arquitecto y figura destacada de la planificación medioambiental, reflejó sus principales contribuciones al paisajismo urbano en publicaciones como Cities (1963), The RSVP Cycles: Creative processes in the human environment (1969) o Taking part: A workshop approach to collective creativity (1974), realizadas en colaboración con Anna (Schuman) Halprin, coreógrafa y pionera de la danza contemporánea, cuyo interés por la relación del cuerpo en movimiento con el espacio urbano le llevó a componer piezas e intervenciones como City dance (1960-69, 1976-77), Initiations and transformations, 1971, o Planetary dance (1987). Anna y Lawrence Halprin indagaron con criterios coreográficos la percepción y el sentido de los espacios construidos como espacios comunitarios y de relación social, proponiendo intervenciones orientadas a la reformulación de la experiencia de los mismos en un contexto urbano de creciente segregación y exclusión social.

Lawrence Halprin se había formado en paisajismo bajo la influencia de los inspiradores de la Bauhaus emigrados a EEUU, entre ellos Walter Gropius y Lazlo Moholy-Nagi, quienes junto con el escultor y coreógrafo Oskar Schlemmer y el arquitecto Farkas Molnár, habían revisado las concepciones dominantes sobre el espacio a través de la danza y su potencial para la transformación social. La propia Anna prepara una disertación sobre Danza y arquitectura (19431), basada en la propuesta que Moholy-Nagi ofrecía en The new vision (1939), donde afirmaba que la danza era un medio eficaz para sensibilizar, experimentar y concebir la forma espacial. En esta línea de trabajo, Anna desarrolla proyectos coreográficos orientados hacia la intervención en espacios construidos fuera de escena (Hangar, 1957; The branch dance, 1957; Apartment 6, 1965) y en espacios públicos, donde el paisaje urbano, la arquitectura y el movimiento ciudadano interactúan con la coreografía, llegando a hacer indiscernibles los frágiles límites que a partir de ese momento pudieran establecerse entre el gesto danzado y el gesto cotidiano (Ceremony of us, 1969; Blank placard dance, 1970; Circle the mountain, 1985). Los experimentos coreográficos de Anna Halprin supusieron un impulso a la renovación de los postulados de la danza moderna, en los que participó un nutrido grupo de artistas multidisciplinares, como John Cage, Merce Cunningham, Robert Morris, o el grupo del que surgirá la Judson Dance Theater (Trisha Brown, Yvonne Rainer, Simone Forti), referentes de la danza postmoderna y el contact improvisation, una técnica de composición coreográfica abierta, basada en la performatividad de las interacciones indeterminadas del cuerpo con otros cuerpos en el espacio físico y social. 


\section{EL GIRO ESPACIAL Y LA PRODUCCIÓN DEL ESPACIO}

Por su parte, la vinculación de Lawrence Halprin con el movimiento moderno en arquitectura respondía a un planteamiento holístico e integral del diseño urbano, comprometido con "las necesidades vitales arquetípicas del ser humano, como individuo y como organización social" (Halprin 1995), en un momento de declive de los principios racionalistas que condujo a la disolución de los CIAM en 1959, junto con el surgimiento de nuevas formas de pensar, experimentar e investigar el espacio, en lo que se ha denominado el spatial turn. El giro, en el ámbito de las humanidades, implicaba cierto grado de retrospección para reflexionar y ejercer una mirada crítica hacia los sesgos culturales y sociales que habían orientado históricamente la construcción de la epistemología del espacio en las diferentes disciplinas (Warf \& Arias 2009), produciendo nuevos enfoques interdisciplinares, como la geo-historia de Fernand Braudel (1963), la geo-filosofía de Gilles Deleuze y Félix Guattari (1991), la tópica expandida de la espacialidad (Soja 2000), o la semiosfera en la que se desenvuelven las relaciones del sujeto con el espacio y con el sentido de la experiencia urbana (Góngora 2012, 29). Como consecuencia del giro epistemológico del espacio, la forma urbana comienza a ser analizada como el resultado de actores en conflicto, donde las formas percibidas representan tan solo un momento de estabilización provisional de la misma (Thom 1977), una especie de tregua inestable permanentemente amenazada por tensiones entre la forma de experimentar y percibir el espacio a través de una gran diversidad de prácticas socio-espaciales, las formas normativas de planificar e intervenir el espacio por parte de las disciplinas urbanísticas, y la apropiación de los espacios por parte de los ciudadanos (Lefebvre [1974] 2013, 97), quienes ensayan constantemente posibilidades alternativas de practicar y transformar el espacio urbano efectivamente construido (Sennet 1970).

\section{LA EXPERIENCIA PARTICIPATIVA DEL ESPACIO URBANO}

Este es el triple escenario conflictual en el que se desenvuelven las propuestas coreo-paisajísticas de los Halprin, recogidas en Taking part: A workshop approach to collective creativity (Fig.1), un proyecto resultante del proceso de 
experimentación interdisciplinar que comienza con Experiments in environments (1966-71), y desemboca en la elaboración de una metodología específica de creación, no solo artística, sino aplicable también a la planificación urbana y a la intervención social en el espacio, denominada RSVP Cycles (1969).

Los Experiments in environments (Fig.2) consistían en talleres interdisciplinares integrados por grupos de estudiantes de arquitectura, diseño, coreógrafos y bailarines, junto con artistas plásticos y miembros de la comunidad que participaban en ensayos de sensibilización ambiental ${ }^{2}$. La exploración del espacio se iniciaba con la realización de itinerarios multisensoriales con los que se trataba de ampliar la experiencia perceptiva de un determinado lugar, propiciando una reflexión acerca de las cualidades del movimiento cotidiano y su relación con el espacio construido. A lo largo de estas actividades de inmersión urbana, los participantes debían tomar conciencia de la acción en sí y del modo en que el cuerpo entra en relación con el espacio construido produciendo una chorea o movimiento en común, cuya cualidad cinética presenta los síntomas de las formas de habitar y transitar los espacios. Los itinerarios urbanos estaban orientados por guías de actuación abiertas a los acontecimientos del entorno, denominadas partituras o scores, que constituirán la base de la metodología RSVP Cycles. Con todo, los experimentos no se agotaban con la investigación cinestésica del medio urbano, sino que trataban también de profundizar en el conocimiento de la problemática social del entorno. Este era precisamente el propósito de Ceremony of us (1969), un proyecto coreográfico de Anna Halprin en el que la diversidad socio-cultural, los diferentes estilos de vida y valores fueron incorporados al enfoque experimental con el propósito de indagar las tensiones y conflictos asociados a la segregación y a la exclusión social, mediante la experiencia del movimiento, del ritmo y de la danza.

\section{COREOGRAFÍA Y RITMOANÁLISIS}

En La producción del espacio, Henri Lefebvre anticipaba la noción de ritmo como un ingrediente de la definición del espacio a la que se incorpora la temporalidad: "El ritmo envuelve lugares [...] y porta en él su ley, su regularidad" (Lefebvre [1974] 2013, 250). La práctica social se compone de ritmos cotidianos que albergan regularidades susceptibles de un ritmo-análisis (Lefebvre, 
[1992] 2004), el cual, más allá de lo inmediatamente perceptible a través de los sentidos, implica un denso entretejido de ritmos biológicos, psicológicos y sociales que regulan la experiencia urbana.

Los experimentos coreo-urbanos de los Halprin retoman esta idea de ritmoanálisis con el fin de diseñar e intervenir en espacios en los que la re-sensibilización de la experiencia cotidiana de los lugares constituye una de las perspectivas críticas del encuentro entre las prácticas artísticas y ciudadanas a las que alude Jacques Rancière cuando advierte que, es mediante esa sensibilización del espacio, de sus ritmos, flujos e interferencias, donde surge la posibilidad de reconfiguración de lo que denomina un reparto de lo sensible, del que no duda en señalar su sustrato político ([2000] 2014, 17). Las intervenciones coreo-paisajísticas de los Halprin, en la medida en que contribuían a la ampliación de la experiencia sensible del espacio urbano, retornaban a la escena urbana aquello que había quedado insensibilizado y consecuentemente pasaba desapercibido en la experiencia cotidiana, dando lugar así al pensamiento, al disenso y a la deliberación política de las prescripciones, tensiones y conflictos que subyacen en los ritmos y espacios ciudadanos. En este contexto, no solo habitar, sino también transitar el espacio urbano constituye una suerte de performance que reconfigura a cada paso el proyecto político de la ciudad y el modo de atribuir significado a la experiencia urbana. En este sentido, un trayecto o un determinado itinerario adquiere forma política en la medida en que incorpora, a través del movimiento mismo, de sus posibilidades, prescripciones e interdicciones, todo un repertorio de dispositivos y de entramados discursivos que han quedado inscritos en el cuerpo del sujeto urbano como una guía implícita de las formas apropiadas de utilizar la ciudad. Pasear, ir al trabajo, a la compra, visitar, permanecer en un lugar o descansar en un parque son performances cotidianas con una fuerte carga política en la medida en que ponen en escena los síntomas de las coerciones que determinan cada una de esas acciones. Basta con alterar el comportamiento esperado en alguno de esos dispositivos para que resurja la fuerza política del diseño urbano y sus efectos y afecciones en el cuerpo ciudadano.

Los ritmo-análisis de las partituras coreográficas que orientaban los itinerarios urbanos de los Experiments in environments tenían el propósito de hacer surgir ese entramado de coerciones presentes en el espacio público, comprometiendo en ellos un cuerpo resiliente que guarda memoria del aprendizaje de los usos del espacio, así como del orden subyacente en el diseño del mismo. En cualquier caso, no se trataba solamente de analizar los ritmos de las personas, sino también el de las máquinas y artefactos cotidianos con los que se 
acompasa el cuerpo y determinan la vida ciudadana. Esta es la idea de dispositivo que recorre desde Foucault (1978) y Agamben (2006) hasta Lefebvre y Halprin, cuando el primero afirma que el conductor de locomotoras no deja de serlo en el momento en que abandona la máquina, sino que ésta permanece incorporada en su vida cotidiana a través de los horarios, los tránsitos, las rutinas y las prácticas discursivas en que participa y lo acompañan.

Mediante los Experiments in environments, los Halprin ponían a prueba el modo en que, incluso los bailarines, cuando tratan de producir un movimiento danzado, establecen una especie de conversación entre la virtualidad o potencias del cuerpo y el marco discursivo en el que se desarrolla la práctica de la danza, convocando a esa conversación tradiciones, teorías, técnicas, espacios y figuras espectatoriales diversas. La práctica de la danza no solo responde entonces al diseño de coreografías explícitas, notaciones, partituras u otras textualidades coreográficas, sino a una amplia red de dispositivos, entre ellos los dispositivos espacio-temporales encarnados en el cuerpo de los bailarines, como un lenguaje que habla de la configuración de esos cronotopos a través de las rutinas (ritornelos) de la vida cotidiana (Deleuze \& Guattari [1972] 2002, 317).

No es por ello extraño que, en una época sumida en un ambiente de fuerte contestación contracultural, surgieran nuevas concepciones experimentales de la danza orientadas hacia la socialización y democratización de la experiencia coreográfica, las cuales al mismo tiempo que ponían en cuestión las nociones de cuerpo, espacio escénico, movimiento y discurso, ponían también de relieve su carácter político desde posiciones como el teatro físico, el contact improvisation, las experiencias interdisciplinares, los happening y site specific, mediante la tentativa de otras formas de relación menos jerárquicas y más participativas del público. De este modo, nuevas alteridades irrumpen con fuerza en la escena de la danza desafiando las formas dominantes de mirar, pensar y participar en la experiencia del movimiento. $Y$ del mismo modo que la danza sale a la calle y se implica en la transformación de las prácticas espaciales cotidianas, arquitectos y urbanistas comienzan a plantear en sus diseños enfoques coreográficos del movimiento, de los flujos y ritmos urbanos, añadiendo a los criterios modernos de usos y funciones, la experiencia corporal y afectiva, semiótica y estética del espacio urbano (Pardo \& Rosales 2012). 


\section{RSVP CYCLES, UNA METODOLOGÍA DE INDAGACIÓN PERFORMATIVA}

Los procesos de trabajo resultantes de los talleres Experiments in environments dieron lugar a la propuesta de los RSVP Cycles, una forma participativa de construir comunidad mediante la sensibilización de los condicionantes espaciales y de las tensiones sociales que surgen en el proceso de trabajo. Así mismo, esta metodología trataba de facilitar la comunicación y la colaboración entre los participantes de diferentes disciplinas, poniendo en cuestión sus respectivas formas de construcción de los objetos de investigación (Bal 2002).

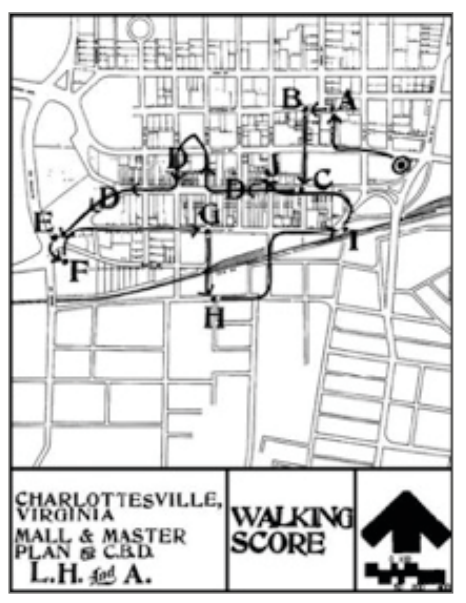

Fig.1 Taking Part Walking Score

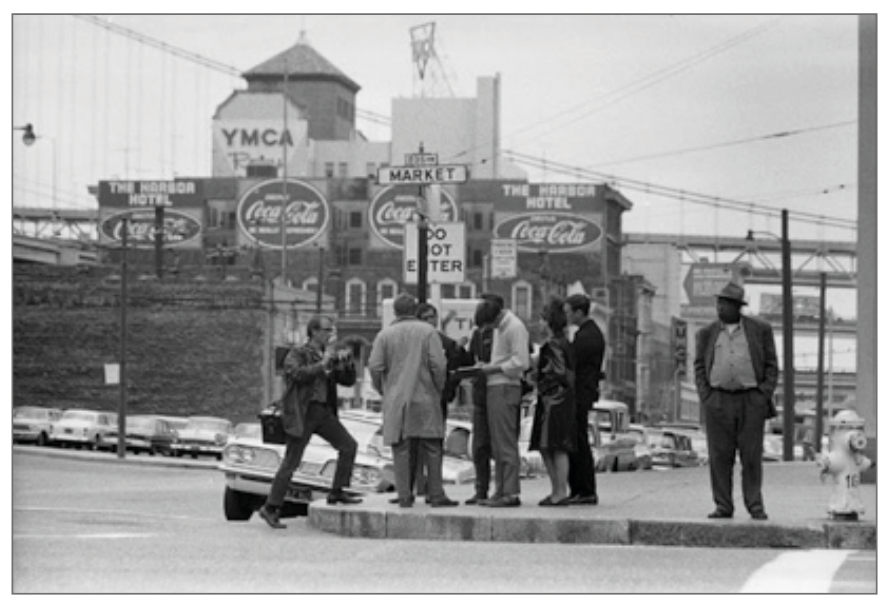

Fig. 2 Market Street Experiment in Environment (1966)

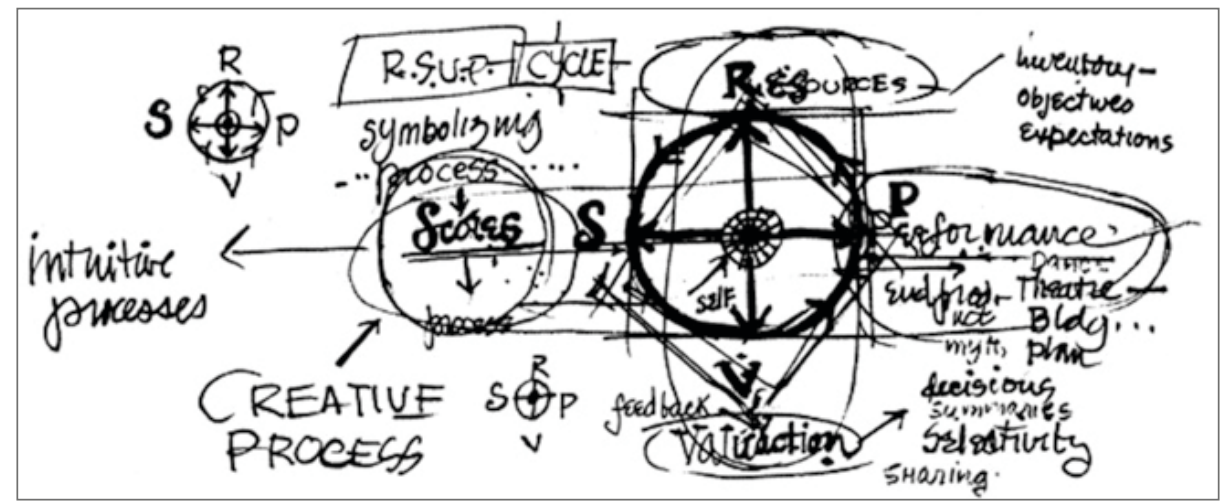

Fig. 3 RSVP Cycles 
RSVP Cycles es un acrónimo de los componentes de una secuencia integrada por (R)ecursos-(S)cores/partituras-e(V)aluación-(P)erformance (Fig.3), con la que se genera un proceso cíclico y participativo de investigación-acción en el espacio construido y comunitario.

El ciclo se inicia con una investigación de los recursos presentes en el entorno, entre los cuales estaban contemplados tanto los materiales objetivos, físicos y perceptuales, como los componentes subjetivos, emocionales y actitudinales que forman la base de la experiencia del espacio, junto con el tejido histórico, social y cultural que envuelve su experiencia.

El segundo componente de la secuencia son las notaciones, partituras o scores (S), destinadas movilizar la experiencia coreográfica del espacio mediante la creación de itinerarios urbanos que incluyen programas de acción a desarrollar en cada situación. El itinerario constituye una forma de recorrer y experimentar la ciudad conectando lugares y escenarios urbanos, y una estrategia destinada a poner en discurso el espacio, cuya notación coreográfica es su forma de expresión. Estas coreografías son a su vez formas de comunicación entre las diversas perspectivas disciplinares que participan en la exploración de la ciudad, así como un proyecto de interacción con el Otro, en tanto que alteridades imprevisibles involucradas en el acontecer urbano. Las partituras devienen así en una suerte de diálogo mediante el cual se trata de dar voz a la complejidad y diversidad de la experiencia urbana. $Y$ dado que estas partituras son abiertas y no tratan de prescribir una coreografía estricta de actuación en el discurrir urbano, constituyen también un modo de focalizar y de hacer visibles los procesos participativos a los que los participantes incorporan sus propias aportaciones, ideas y emociones, posibilitando así la formación de comunidades autotélicas basadas en objetivos comunes surgidos en el curso mismo de la acción.

De este modo, y a diferencia de las guías cerradas que producen las normativas urbanísticas, Lawrence Halprin sugería que era preciso poner el énfasis en la notación de los movimientos cotidianos de la comunidad, a la que denominó motation, cuyo objetivo consistía en hacer sensibles y visibles todos los aspectos del proceso, en todo momento, para poner en cuestión tanto el espacio normativo, como el reparto de lo sensible y de las competencias para actuar en un determinado espacio social, al que se refería Rancière. En este sentido, el espacio sensible de la experiencia urbana no se refiere únicamente a las cualidades físicas y sensoriales del mismo, sino a la atribución de competencias -e incompetencias- para actuar en él, de tal forma que en la configuración 
misma del espacio estarían inscritas las condiciones de sus usos y funciones como si se tratara del diseño de una dramaturgia inserta en la escenografía urbana. Las partituras de los RSVP Cycles tenían como función la movilización de exploraciones alternativas de esas escenografías construidas, con el fin de hacer evidente la organización social y las relaciones de poder para las que habían sido dispuestas.

La valuación $(V)$ es un neologismo acuñado para señalar la necesidad de un análisis del principio conflictual constitutivo de la forma urbana y de su subjetivación a través de los hábitos, ritmos y dispositivos que regulan la experiencia urbana, todo ello con el fin de promover el debate, la retroalimentación y la toma de decisiones acerca de las acciones y performances a realizar en la fase siguiente del ciclo RSVP. De este modo, se trataba de someter las partituras coreográficas a una reflexión constante para facilitar su adecuación a las condiciones imprevisibles y cambiantes del proceso. Así, mientras la partitura movilizaba la exploración sensorial del entorno y la identificación de los recursos presentes en el mismo, la (e)valuación incorpora los valores implicados en el proceso. No se trata por tanto de producir únicamente un conocimiento situado del espacio, ni una mera comprensión de las tensiones y conflictos que lo atraviesan, sino de la incorporación de éstos al proceso de investigación performativa orientado a la acción e intervención en dichos espacios.

Finalmente, la performance o actuación $(\mathrm{P})$ con la que se cierra el ciclo, constituye la realización de la partitura y proporciona un estilo particular al proceso. En las performances el ciclo opera en dos niveles íntimamente relacionados. El ciclo interno orientado a la toma de conciencia sensorial y a la realización de una experiencia personal y singular de los espacios; y el ciclo exterior, comunitario, orientado al grupo y a la experiencia colectiva de encuentro con el Otro a través de la indagación del devenir de la vida cotidiana que se desarrolla en dichos espacios.

Los RSVP Cycles proporcionan, en definitiva, una metodología de trabajo para la experimentación, la investigación y el conocimiento situado de la vida en los espacios urbanos, además de una propedéutica para la intervención abierta a las contingencias de lo cotidiano confrontada con las determinaciones inscritas en los mismos. 


\section{Referencias bibliográficas}

Agamben, Giorgio. (2006) 2015. ¿Qué es un dispositivo?. Traducción de Mercedes Ruvituso. Barcelona: Anagrama

Bal, Mieke. (2002) 2009. Conceptos viajeros en las humanidades. Traducción, Yaiza Hernández Velázquez. Murcia: Cendeac

Braudel, Fernand. (1963) 1995. A history of civilizations. Translated by Richard Mayne. London: Penguin Books

Deleuze, Gilles \& Félix Guattari. (1972) 2002. Mil mesetas: Capitalismo y esquizofrenia. Traducción de José Vázquez Pérez con la colaboración de Umbelina Larraceleta. Valencia: Pre-textos

Deleuze, Gilles \& Félix Guattari. 1991. Qu'est-ce que la philosophie?. París: Minuit

Foucault, Michel. (1978) 1991. "El juego de Michel Foucault". En Saber y verdad, trad., Julia Varela \& Fernando Álvarez-Uría, 127-62. Madrid: La Piqueta

Foucault, Michel. (1988) 2015. Dits et écris 1954-1988: II, 1976-1988. Éd. établie sous la dir. de D. Defert et F. Ewald avec la coll. de J. Lagrange. París: Gallimard

Góngora Villabona, Lizardo Álvaro. 2012. "Semiótica del paisaje urbano". En Semióticas urbanas: Espacios simbólicos, Neyla Graciela Pardo Abril \& Horacio Rosales Cueva, coords. Buenos Aires: La Crujía

Halprin, Lawrence \& Jim Burns. 1974. Taking part: A workshop approach to collective creativity. With contributions by Anna Halprin \& Paul Baum. Cambridge MA: MIT

Halprin, Lawrence. 1969. The RSVP cycles: Creative processes in the human environment. New York: George Braziller

Halprin, Lawrence. 1995. "Invisible gardens: The search for modernism in the American landscape". Review of the book of Peter Walker \& Melanie Simo (1994). Choice Reviews Online 32(07)

Lefebvre, Henri (1974) 2013. La producción del espacio. Prólogo de lon Martínez Lorea; introducción y traducción de Emilio Martínez Gutiérrez. Madrid: Capitán Swing

Lefebvre, Henri. (1992) 2004. Ritmoanálisis: Espacio, tiempo y vida cotidiana. Traducido [al inglés] por Stuart Elden \& Gerald Moore; con una introd. de Stuart Elden; trad. [al español] por Consuelo Berrios. www.antropologia.cat/estatic/files/Ritmoanalisis_Lefebvre.pdf

Merriman, Peter. 2010. "Architecture/dance: Choreographing and inhabiting spaces with Anna and Lawrence Halprin". Cultural Geographies 17(4): 427-49

Pardo Abril, Neyla Graciela \& Horacio Rosales Cueva, coords. 2012. Semióticas urbanas: Espacios simbólicos. Buenos Aires: La Crujía

Rancière, Jacques. (2000) 2014. El reparto de lo sensible: Estética y política. Cuidado de la edición Magalí C. Álvarez Howlin. Buenos Aires: Prometeo

Sennet, Richard. (1970) 2002. Vida urbana e identidad personal. Traducción de Josep Rovira; prólogo de Tomàs Llorens. Barcelona: Península

Soja, Edward W. 2000. Postmetrópolis: Critical studies of cities and regions. Oxford: Blackwell

Thom, René. (1977) 1980. Stabilitá structurale e morfogenes: Saggio di una teoría generale deo modelli. Traduzione di Antonio Pedrini. Torino: Einaudi 
Warf, Barney \& Santa Arias, eds. 2009. The spatial turn: Interdisciplinary perspectives. London: Routledge

Notas

1 "Dance and architecture". MPD AH Archives (citado en Merriman 2010)

${ }^{2}$ Diversas sesiones de los Experiments in Environments se hallan profusamente documentadas en The Architectural Archives, de la Universidad de Pensilvania: https://www.design. upenn.edu/architectural-archives/digital-resources. 